\title{
An Integral Equation Representation for Overlapping Generations in Continuous Time
}

\author{
Chris Edmond*
}

First draft: January 2005. This draft: November 2007.

\begin{abstract}
This paper develops a method for solving for the dynamic general equilibrium of a deterministic continuous time overlapping generations model with a finite-horizon life-cycle. The model has isoelastic preferences and allows for general assumptions about individual endowments and demographics. Solving for an equilibrium reduces to solving a nonlinear integral equation. In the special case of log utility, the integral equation is linear and global approximations to a solution are easily computed with linear algebra.
\end{abstract}

Keywords: overlapping generations, continuous time, life-cycle, intertemporal prices.

JEL classifications: D5, D9, E2.

The overlapping generations model is one of the workhorses of modern macroeconomics. This paper outlines a continuous time OLG model where agents live for a given finite interval of time so that genuine life-cycle behavior is possible. ${ }^{1}$

In applied macroeconomics, OLG models are set in discrete time and individuals have many decision periods per lifetime (Auerbach and Kotlikoff, 1987; Ríos-Rull, 1996). Long-lived models of this kind are attractive because they allow for realistic timing conventions and an intuitive calibration to annual or quarterly data. In practice, however, long-lived discrete time models are difficult to work with computationally. The main advantage of the continuous time model outlined below is that it simultaneously permits agents to have many decisions per lifetime but is computationally simple.

The model is presented in Section 1 and allows for isoelastic utility and general assumptions about individual endowments (and demographics). The main result of this paper is given in Section 2 which shows how to represent the equilibrium fixed point problem as a specific, generally nonlinear, integral equation which needs to be solved for an unknown intertemporal price function.

*Department of Economics, Stern School of Business, New York University. Email: 〈cedmond@stern.nyu.edu〉. I thank Costas Azariadis for his continued enthusiasm and advice. I am also grateful to Jess Benhabib, Roger Farmer, Herakles Polemarchakis and seminar participants at UCLA, the University of Melbourne and NYU for comments.

${ }^{1}$ Following Yaari (1965), Blanchard (1985) studies a continuous time OLG model where agents die at an exogenously given exponential rate. This simplifies the analysis considerably but also excludes life-cycle behavior. Weil (1989) studies a similar model of overlapping families with infinite horizons. Other related literature is discussed at the end of the paper. 
Section 3 shows for the special case of log utility that this integral equation is linear and solving for an equilibrium involves finding intertemporal prices $p(t)$ satisfying:

$$
p(t)=\int_{0}^{\infty} p(s) k(t, s) d s+f(t)
$$

for time $t \in[0, \infty)$. In this equation the integral kernel $k(t, s)$ and forcing process $f(t)$ are known functions of primitives of the economy, including individual endowments and the initial asset distribution. Although this problem generally does not have a closed form solution, there are well known algorithms for finding global approximations to its solution (Atkinson, 1997; Baker, 1977; Judd, 1998; Press, Teukolsky, Vetterling, and Flannery, 1992). These methods exploit the analogy between the linear integral equation and finite dimensional linear algebra. The case of general isoelastic utility leads to a similar (but nonlinear) integral equation that, as discussed in Section 4, is also computationally tractable — no matter how long-lived individuals are.

\section{Model}

Consider a deterministic endowment economy populated by an infinity of overlapping generations of agents. Time is continuous and indexed by $t \in[0, \infty)$.

Demographics. The economy is populated by two kinds of agents. First, at each instant of time $t>0$ a continuum of identical agents are born and live for a finite period of time, $l>0$. Second, at date $t=0$ there is a pre-existing mass of agents. Since agents have a lifetime of $l$, at date $t=0$ we need to account for all the generations 'born' in the interval $(-l, 0]$. These transitional generations play the role of the 'initial old' in the classic two-period overlapping generations model of Diamond (1965). A transitional generation $v \in(-l, 0]$ lives over $t \in[0, v+l)$. Let the set of all generations alive at date $t \geq 0$ be $\mathcal{G}(t):=(t-l, t]$. Also, let the set of dates over which generation $v \in(-l, \infty)$ lives be $\mathcal{A}(v):=\{t \geq 0 \mid \max [0, v] \leq t<v+l\}$. For $v \geq 0$ this is just $[t, t+l)$. To simplify notation, let the total population be constant (and normalized to size one) ${ }^{2}$

Endowments. Each instant $t$ generation $v$ is endowed with an exogenous amount of a single nonstorable consumption good $y(t, v) \geq 0$. No endowment is received if the agent is not alive: $y(t, v)=$ 0 for all $(t, v) \notin \mathcal{A}(v) \times \mathcal{G}(t)$. Let $Y(t):=\int_{\mathcal{G}(t)} y(t, v) d v>0$ denote the aggregate endowment at $t$ and let $\varphi(t, v):=y(t, v) / Y(t) \geq 0$ denote the density of generation $v$ 's endowment.

In addition to physical endowments, transitional generations are endowed with assets - preexisting claims to consumption. Let $a(0, v)$ denote the net assets of generation $v$ at time $t=0$. For generations $v>0$ these are zero. For transitional generations $v \in \mathcal{G}(0)$ these may be positive or negative but must net out to zero, $\int_{\mathcal{G}(0)} a(0, v) d v=0$. There is no outside asset.

\footnotetext{
${ }^{2}$ Appendix A generalizes the model to allow for aggregate population growth, changes in the relative proportions of young and old, and within-cohort endowment heterogeneity.
} 
Preferences and budget constraints. Each individual has preferences over dated consumption goods represented by a time-seperable utility function with isoelastic instantaneous utility and exponential discounting:

$$
\int_{\mathcal{A}(v)} e^{-\rho(t-v)} u[c(t, v)] d t, \quad u(c):=\frac{c^{1-\sigma}-1}{1-\sigma}, \quad \rho \geq 0, \quad \sigma>0
$$

For $t \in \mathcal{A}(v)$ the flow constraint facing an individual is:

$$
\dot{a}(t, v)=r(t) a(t, v)+y(t, v)-c(t, v)
$$

where a dot denotes differentiation with respect to time and with given initial conditions $a(v, v)$ and instantaneous interest rate $r(t)$.

We can integrate the flow constraint to get each individual's intertemporal budget constraint:

$$
\int_{\mathcal{A}(v)} p(t) c(t, v) d t=\int_{\mathcal{A}(v)} p(t) y(t, v) d t+p(v) a(v, v)
$$

where $p(t)$ is the intertemporal price of consumption, $\dot{p}(t) / p(t)=:-r(t)$. Using $y(t, v)=0$ for all $(t, v) \notin \mathcal{A}(v) \times \mathcal{G}(t)$ and $a(v, v)=0$ for all $v>0$, the right-hand side of the intertemporal constraint can be written:

$$
W(p, v):=\int_{0}^{\infty} p(s) y(s, v) d s+p(0) a(0, v)
$$

where $W(p, v)$ denotes an individual's intertemporal wealth as of date $t=0$ given prices $p$.

Optimization. Taking prices as given, each individual chooses consumption $c(t, v) \geq 0$ to maximize utility (1) subject to their intertemporal constaint (3). This a concave programming problem over a convex constraint set. The first order condition characterizing consumption is:

$$
e^{-\rho(t-v)} c(t, v)^{-\sigma}=\lambda(v) p(t)
$$

where $\lambda(v) \geq 0$ is the time-invariant Lagrange multiplier on the intertemporal budget constraint of generation $v$. Differentiating both sides of (5) with respect to $t$ gives the standard consumption Euler equation $\dot{c}(t, v) / c(t, v)=(r(t)-\rho) / \sigma$.

Using the intertemporal constraint, the solution for the Lagrange multiplier is:

$$
\lambda_{\sigma}(p, v):=\left[\int_{\mathcal{A}(v)} e^{-\frac{\rho}{\sigma}(s-v)} p(s)^{\frac{\sigma-1}{\sigma}} d s\right]^{\sigma} W(p, v)^{-\sigma}
$$

Plugging this solution for the multiplier into (5) gives the consumption function:

$$
c_{\sigma}(p, t, v):=\alpha_{\sigma}(p, t, v) \frac{W(p, v)}{p(t)}
$$

where $\alpha_{\sigma}(p, t, v)$ denotes the expenditure shares:

$$
\alpha_{\sigma}(p, t, v):=\frac{e^{-\frac{\rho}{\sigma} t} p(t)^{\frac{\sigma-1}{\sigma}}}{\int_{\mathcal{A}(v)} e^{-\frac{\rho}{\sigma} s} p(s)^{\frac{\sigma-1}{\sigma}} d s}
$$

In the special case of $\log$ utility $(\sigma=1)$, we have the familiar property that expenditure shares do not depend on prices $p$. 
Equilibrium. An equilibrium is feasible consumption $c \geq 0$ and a price $p>0$ such that (i) taking as given prices, each individual chooses consumption to maximize utility, and (ii) markets clear:

$$
\int_{\mathcal{G}(t)} c_{\sigma}(p, t, v) d v=\int_{\mathcal{G}(t)} y(t, v) d v=: Y(t)
$$

The aggregate endowment $Y(t)>0$ is exogenous, so we know the right-hand side of (9). We need to find a function $p$ that ensures that markets clear on all dates. That is, we have to solve a nonlinear integral equation for $p$. The market clearing condition gives us an integral equation of the 'first kind,' meaning that the unknown function $p$ enters only inside the integral. In general finding numerical solutions of first kind equations is harder that finding solutions of 'second kind' integral equations where the unknown function also enters outside the integral operator (Atkinson, 1997; Press, Teukolsky, Vetterling, and Flannery, 1992). ${ }^{3}$ In Section 2 below, the properties of the consumption function (7) are used to rewrite (9) as a second kind integral equation.

Because the consumption function is zero degree homogeneous in prices, if $p$ is an equilibrium price, so is $\xi p$ for any scalar $\xi>0$. We can only determine relative prices. Let $p(0)=1$ be the normalization.

Benchmark and the role of compositional effects. Since each individual satisfies their consumption Euler equation, they all have the same consumption growth. But in general this does not mean the growth of the aggregate endowment satisfies a corresponding 'aggregate' Euler equation. In general $\dot{Y}(t) / Y(t) \neq(r(t)-\rho) / \sigma$. To see this, multiply both sides of the Euler equation by $c(t, v)$ and integrate both sides over $\mathcal{G}(t)$ to get:

$$
\int_{\mathcal{G}(t)} \dot{c}(t, v) d v=\frac{r(t)-\rho}{\sigma} \int_{\mathcal{G}(t)} c(t, v) d v=\frac{r(t)-\rho}{\sigma} Y(t)
$$

But using Leibniz's rule:

$$
\begin{aligned}
\dot{Y}(t)=\frac{d}{d t} \int_{\mathcal{G}(t)} c(t, v) d v & =c(t, t)-c(t, t-l)+\int_{\mathcal{G}(t)} \dot{c}(t, v) d v \\
& =c(t, t)-c(t, t-l)+\frac{r(t)-\rho}{\sigma} Y(t)
\end{aligned}
$$

Only if the consumption of the very young and very old is the same, $c(t, t)=c(t, t-l)$, does this reduce to the familiar benchmark $\dot{Y}(t) / Y(t)=(r(t)-\rho) / \sigma$ in which case the equilibrium price function is simply $\log [p(t)]=-\rho t-\sigma \int_{0}^{t} \gamma(s) d s$ with $\gamma(t):=\dot{Y}(t) / Y(t)$ the instantaneous growth rate of the aggregate endowment. See Blanchard (1985) or Weil (1989) for further discussion.

\section{Integral equation representation of equilibrium prices}

The main contribution of this paper is a simple functional equation representation of the equilibrium fixed point problem. Intertemporal prices $p$ solve a fixed point problem of the form $p=T_{\sigma} p$ where $T_{\sigma}$ is defined by a nonlinear integral operator.

\footnotetext{
${ }^{3}$ If the right-hand side of (9) is smooth then the unknown function $p$ has to be something that when integrated gives a smooth answer. But lots of badly behaved functions are smooth after they have been integrated. See Press, Teukolsky, Vetterling, and Flannery (1992) for detailed discussion of these ill-posed inversion problems.
} 
PROPOSITION 1. Intertemporal prices $p$ solve the nonlinear integral equation:

$$
p(t)=\int_{0}^{\infty} p(s) k_{\sigma}(p, t, s) d s+f_{\sigma}(p, t)
$$

where:

$$
\begin{aligned}
k_{\sigma}(p, t, s) & :=\int_{\mathcal{G}(t)} \alpha_{\sigma}(p, t, v) \frac{y(s, v)}{Y(t)} d v \\
f_{\sigma}(p, t) & :=\int_{\mathcal{G}(t)} \alpha_{\sigma}(p, t, v) \frac{a(0, v)}{Y(t)} d v
\end{aligned}
$$

and where the expenditure shares $\alpha_{\sigma}(p, t, v)$ are given by $(8)$.

Proof. Substitute the consumption function $c_{\sigma}(p, t, v)$ from equation $(7)$ into the market clearing conditions (9) and multiply both sides by $p(t)$ to get:

$$
p(t) Y(t)=\int_{\mathcal{G}(t)} \alpha_{\sigma}(p, t, v) W(p, v) d v
$$

Now substitute in the definition of intertemporal wealth:

$$
p(t) Y(t)=\int_{\mathcal{G}(t)} \alpha_{\sigma}(p, t, v)\left[\int_{0}^{\infty} p(s) y(s, v) d s+p(0) a(0, v)\right] d v
$$

And change the order of integration for the double integral on the right-hand side of this expression (for this calculation, $t$ is taken as a parameter) to get:

$$
p(t) Y(t)=\int_{0}^{\infty} \int_{\mathcal{G}(t)} \alpha_{\sigma}(p, t, v) p(s) y(s, v) d v d s+p(0) \int_{\mathcal{G}(t)} \alpha_{\sigma}(p, t, v) a(0, v) d v
$$

If we divide both sides by $Y(t)>0$ and define $k_{\sigma}(p, t, s)$ and $f_{\sigma}(p, t)$ as in equations (11)-(12), then we have the desired representation.

Equation (10) is a nonlinear Urysohn integral equation (Baker, 1977). The right hand side of (10) defines an operator $T_{\sigma}$ that takes prices as an argument:

$$
\left(T_{\sigma} p\right)(t):=\int_{0}^{\infty} k_{\sigma}(p, t, s) d s+f_{\sigma}(p, t)
$$

Equilibrium prices are fixed points of this operator. In practice, approximations to equilibrium prices can be obtained by iterating on $T_{\sigma}$, as outlined below.

\section{Solving the model with log utility}

In the special case of $\log$ utility, the operator $T_{\sigma}$ is linear. This is interesting in its own right, but also, because it makes the log case especially easy to solve, helps provide a 'good' initial condition for approximate solutions of the general problem computed by iterating on $T_{\sigma}$. 


\subsection{Linear integral equation}

As $\sigma \rightarrow 1$ the expenditure shares no longer depend on $p$ and we have:

$$
\lim _{\sigma \rightarrow 1} \alpha_{\sigma}(p, t, v)=\frac{e^{-\rho t}}{\int_{\mathcal{A}(v)} e^{-\rho s} d s}=: \alpha(t, v)
$$

For generations $v \geq 0$ expenditure shares are proportional to $e^{-\rho(t-v)}$ with a constant of proportionality ensuring they integrate to 1 over a lifetime of length $l$. We now have:

$$
\begin{aligned}
\lim _{\sigma \rightarrow 1} k_{\sigma}(p, t, s) & =\int_{\mathcal{G}(t)} \alpha(t, v) \frac{y(s, v)}{Y(t)} d v=: k(t, s) \\
\lim _{\sigma \rightarrow 1} f_{\sigma}(p, t) & =\int_{\mathcal{G}(t)} \alpha(t, v) \frac{a(0, v)}{Y(t)} d v=: f(t)
\end{aligned}
$$

both independent of prices. So the nonlinear Urysohn integral equation (10) becomes:

$$
p(t)=\int_{0}^{\infty} p(s) k(t, s) d s+f(t)
$$

This is a linear Fredholm equation of the second kind (Atkinson, 1997; Baker, 1977; Judd, 1998; Press, Teukolsky, Vetterling, and Flannery, 1992; Tricomi, 1957). ${ }^{4}$ Equilibrium prices are fixed points $p=T p$ of the linear operator $T:=\lim _{\sigma \rightarrow 1} T_{\sigma}$.

Discussion. Let $K$ denote the linear integral operator implied by the kernel function $k(t, s)$ so that equilibrium prices solve $p=T p=K p+f$. Then if the resolvent operator $R=: \sum_{i=0}^{\infty} K^{i}$ exists, we have:

$$
p=(I-K)^{-1} f=R f=f+K R f
$$

A standard sufficient condition for the resolvent operator to exist is that the operator norm $\|K\|<$ 1 (Atkinson, 1997; Tricomi, 1957). Intuitively, this ensures $\sum_{i=0}^{\infty} K^{i}$ converges to a well behaved limiting operator.

The properties of equilibrium prices are determined by the known integral kernel function $k(t, s)$ and the known forcing process $f(t)$.

Integral kernel. The integral kernel $k(t, s)$ encodes information about the (appropriately discounted) relative scarcity of consumption goods at different dates $(t, s)$. Since $y(t, v)=0$ for all $(t, v) \notin \mathcal{A}(v) \times \mathcal{G}(t)$, the integral kernel can be rewritten:

$$
k(t, s)= \begin{cases}0 & \text { if } s<t-l \\ k_{-}(t, s) & \text { if } t-l \leq s<t \\ k_{+}(t, s) & \text { if } t \leq s \leq t+l \\ 0 & \text { if } s>t+l\end{cases}
$$

\footnotetext{
${ }^{4}$ Many macroeconomists will be familiar with linear Fredholm integral equations of the second kind. A leading example is the Lucas (1978) asset pricing model where the unknown function is the price of the single non-storable consumption good in a given state where the state follows an exogenous Markov process with continuous support. See Tauchen and Hussey (1991).
} 
where

$$
\begin{array}{ll}
k_{-}(t, s):=\int_{\max \{0, t-l\}}^{s} \alpha(t, v) \frac{y(s, v)}{Y(t)} d v, \quad t-l \leq s<t \\
k_{+}(t, s):=\int_{\max \{0, s-l\}}^{t} \alpha(t, v) \frac{y(s, v)}{Y(t)} d v, & t \leq s \leq t+l
\end{array}
$$

Forcing process. The know forcing process $f(t)$ inherits its key properties from the distribution of initial assets $a(0, v)$. In particular, because $a(0, v)=0$ for $v>0$, we also have $f(t)=0$ for $t>l$. That is, for $t>l$ there are no more living transitional generations and their direct influence on current prices is exactly zero. Of course, the transitional generations continue to have an indirect effect. The first round of indirect effects takes place because the forcing process at (say) the point $t=l / 2$ directly affects the price at $t$ and indirectly affects all the prices on dates $[0, l / 2+l]$ through the kernel. There are also a whole collection of higher order rounds of indirect effects so the value of the forcing function at any date $t$ matters for the whole price function.

Example: balanced growth. Let the aggregate endowment grow at rate $\gamma \geq 0$ so that $Y(t)=e^{\gamma t}$ and let the endowment be uniformly distributed with $\varphi(t, v)=1 / l$ for all $(t, v) \in \mathcal{A}(v) \times \mathcal{G}(t)$. Then for $t>l$ so that we are removed from the influence of the transitional generations:

$$
\begin{aligned}
& k_{-}(t, s)=\beta e^{(\gamma+\rho)(s-t)}\left[1-e^{-\rho(s-t+l)}\right] \\
& k_{+}(t, s)=\beta e^{\gamma(s-t)}\left[1-e^{-\rho(t-s+l)}\right]
\end{aligned}
$$

where $\beta:=1 /\left[\left(1-e^{-\rho l}\right) l\right]$. Notice that $k_{-}$is increasing in $s$ and that $k_{+}$is decreasing in $s$ and $k_{-}(t, t)=k_{+}(t, t)=1 / l$. In short, the kernel is a two-sided smoother around the point $t$ giving most weight to $s$ close to $t$ (and zero weight to those $s$ that are more than $l$ from the current $t$ ). Around $t$ the speed at which the weight given to $s$ falls off depends on the rate of time preference $\rho$ and the growth rate of the aggregate endowment $\gamma$. The weights diminish faster when the rate of time preference is higher.

Given that for any kernel $f(t)=0$ for $t>l$, the integral equation characterizing equilibrium prices can be written:

$$
p(t)=\int_{t-l}^{t+l} p(s) k(t, s) d s, \quad t>l
$$

And with this balanced growth example, from (21)-(22), the integral kernel can be written as a function of $t-s$ only, $k(t, s)=\hat{k}(t-s)$. So (23) provides a simple way of solving for the steady-state price path along which $\dot{p}(t) / p(t)=-r$ for some constant interest rate $r$. We have:

$$
1=\int_{t-l}^{t+1} e^{-r(s-t)} k(t, s) d s=\int_{-l}^{+l} e^{-r z} \hat{k}(z) d z, \quad t>l
$$

which is one equation to be solved for $r$ (there may be several solutions). This interest rate characterizes the limiting equilibrium price path, $p(t) \sim e^{-r t}$ for large $t$, but since $f(t)$ affects 
prices at all $t$, even for this simple example equilibrium prices will not exactly equal $e^{-r t}$ at any $t$. Also, the steady state interest rate $r$ does not generally equal the benchmark $\rho+\gamma$ that would obtain with log utility when there are no compositional effects.

One approach to solving the model numerically would be to use local approximation methods based on perturbing the price path $p(t)$ around $e^{-r t}$. But this neglects the role of transitional generations and makes such solutions unattractive for analyzing both the effects of policy changes along a transition path as well as the full welfare implications of such policy changes. For linear Fredholm integral equations of the second kind, like (19), there are several well known global approximation methods, as outlined below, and so there is no need for local approximations around steady-state.

\subsection{Global approximation of $p(t)$ with log utility}

The two most common global approximation methods for solving a linear integral equation like (19) are Nystrom's extension and the method of approximation by separable kernels. Both methods reduce the integral equation problem to a finite dimensional linear algebra problem. Since these methods are well known in the numerical analysis literature (Atkinson, 1997; Baker, 1977; Judd, 1998; Press, Teukolsky, Vetterling, and Flannery, 1992), the discussion here is deliberately terse.

Nystrom's extension. Let $s_{i}, \omega_{i}$ for $i=1, \ldots, J$ denote a set of $J$ numerical quadrature nodes and weights that allow us to approximate the integral operator on the right hand side of (19) by a finite sum:

$$
\int_{0}^{\infty} p(s) k(t, s) d s \approx \sum_{i=1}^{J} p\left(s_{i}\right) k\left(t, s_{i}\right) \omega_{i}
$$

Then the linear integral equation can be approximated by the finite system of equations:

$$
p_{i}=\sum_{j=1}^{J} p_{j} k_{i j}+f_{i}, \quad i=1, \ldots, J
$$

where in a slight abuse of notation, $p_{i}:=p\left(s_{i}\right), k_{i j}:=k\left(s_{i}, s_{j}\right)$ and $f_{i}:=f\left(s_{i}\right)$. In matrix notation, $\mathbf{p}=\mathbf{K p}+\mathbf{f}$. If $\operatorname{det}(\mathbf{I}-\mathbf{K}) \neq \mathbf{0}$ then there is a unique vector, call it $\hat{\mathbf{p}}$, given by the resolvent matrix $\mathbf{R}=(\mathbf{I}-\mathbf{K})^{\mathbf{- 1}}$ so that $\hat{\mathbf{p}}=\mathbf{R f}=(\mathbf{I}-\mathbf{K})^{-\mathbf{1}} \mathbf{f}$. This gives us approximate equilibrium prices $\hat{p}_{i}$ at the numerical quadrature nodes $s_{i}$, for $i=1, \ldots, J$. Nystrom's extension then gives prices for any $t \in[0, \infty)$ using:

$$
p(t)=\sum_{i=1}^{J} \hat{p}_{i} k\left(t, s_{i}\right) \omega_{i}+f(t)
$$

Separable kernels. If the integral kernel can be written $k(s, t)=\sum_{i=1}^{J} g_{i}(s) h_{i}(t)$ for a finite collection of (known) basis functions $g_{i}(s)$ and $h_{i}(t)$ then it is separable. In this case, the linear integral 
equation (19) reduces exactly to a finite dimensional linear algebra problem and has the solution:

$$
p(t)=\sum_{i=1}^{J} \hat{p}_{i} h_{i}(t)+f(t)
$$

where $\hat{p}_{i}$ for $i=1, \ldots, J$ denote the solutions to the finite system of equations $p_{i}=\sum_{j=1}^{J} p_{j} k_{i j}+f_{i}$ [as in (26)]. And exploiting the separability of the kernel we have exact formulas for the coefficients:

$$
\begin{aligned}
k_{i j} & :=\int_{0}^{\infty} h_{i}(t) g_{i}(t) d t \\
f_{i} & :=\int_{0}^{\infty} h_{i}(t) f(t) d t
\end{aligned}
$$

If the kernel is separable, then (28) gives an exact global solution for the equilibrium price function. In general, the kernel will not be exactly separable but can be arbitrarily well approximated as such $k(s, t) \approx \sum_{i=1}^{J} g_{i}(s) h_{i}(t)$ for large enough $J$ and appropriate basis functions $g_{i}(s)$ and $h_{i}(t)$.

\subsection{Numerical examples}

The following examples were computed using Nystrom's extension. In each case, the lifetime was set to $l=75$ years.

Stationary economy. Let the aggregate endowment be constant and the distribution of individual endowments within the current population be uniform, $\varphi(t, v)=1 / l$. The solid line in Figure 1 is the equilibrium price function when the time discount rate is $\rho=0.05$. The dashed line is the benchmark $e^{-0.05 t}$ which gives the equilibrium prices that would obtain in an analogous representative agent model. The equilibrium real interest rates in this continuous time OLG economy are high. For this example, the initial asset distribution has old agents with positive assets and young agents with negative assets. The top row of Figure 2 shows the initial asset distribution $a(0, v)$, the corresponding forcing process $f(t)$, and again the equilibrium prices $p(t)$. The bottom row of Figure 2 shows the reverse case, when the young have assets and the old have liabilities. The forcing process $f(t)$ obtains much of its shape from key properties of the initial asset distribution, so, for example, when the initial old have positive assets then $f(0)>0$ and $f(t) \rightarrow 0^{-}$as $t \rightarrow l^{-}$. The reverse is true when the old have initial liabilities. In this example, the equilibrium price function is not very sensitive to properties of the forcing process. The dashed lines show the same objects but with a higher discount rate of $\rho=0.10$. This has a much greater influence over the whole price function.

Distributional effects. The top panel of Figure 3 shows the equilibrium price function $p(t)$ for the case of a uniform distribution of individual endowments and for 'hump-shaped' individual endowments that are a quadratic function of age, proportional to $(t-v)(l-t+v)$. The only difference between these economies is the distribution of individual endowments. These different patterns of individual endowments lead to quite pronounced differences in equilibrium prices. 


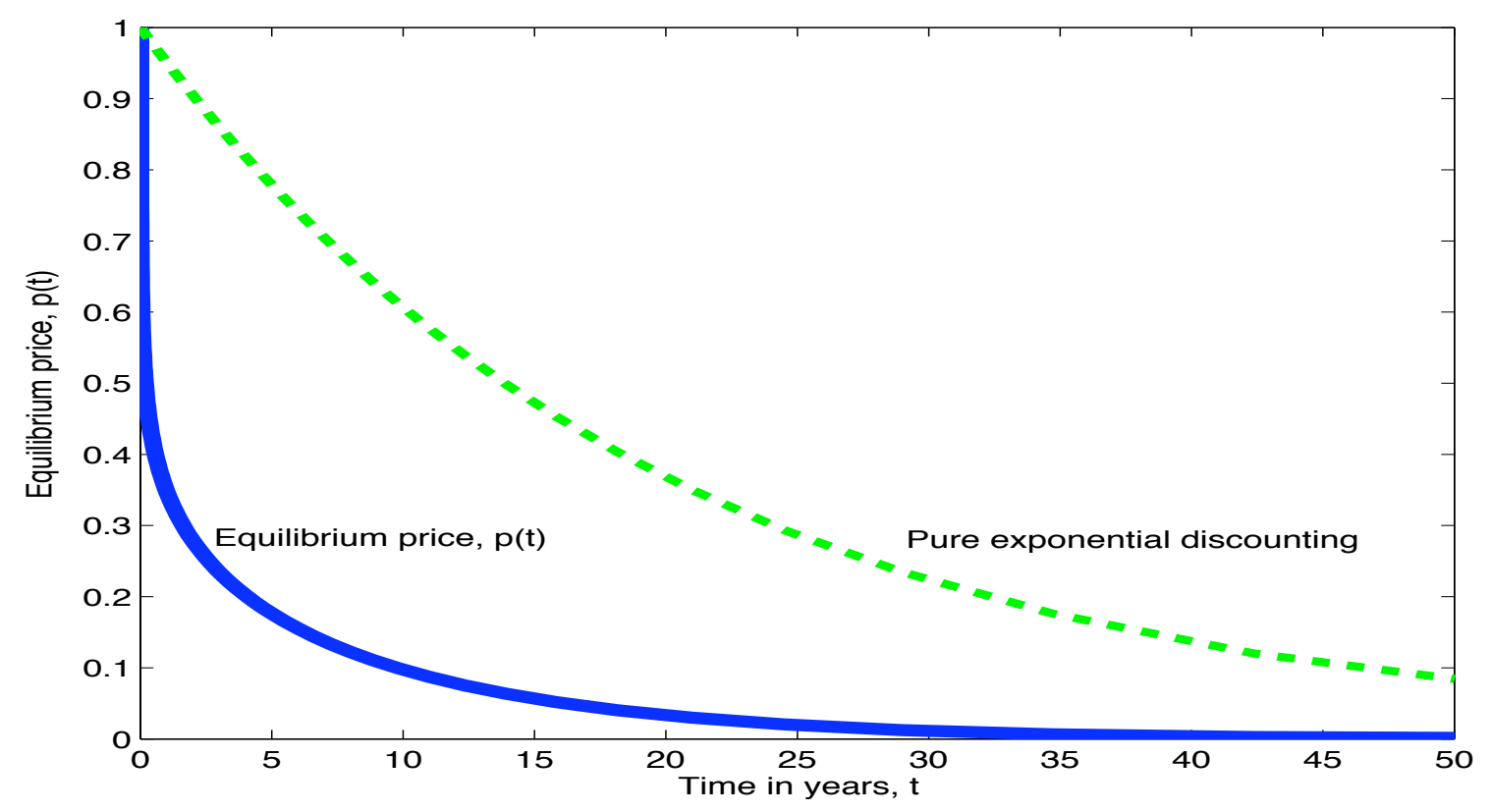

Figure 1: Equilibrium intertemporal prices $p(t)$ versus benchmark $e^{-\rho t}$.
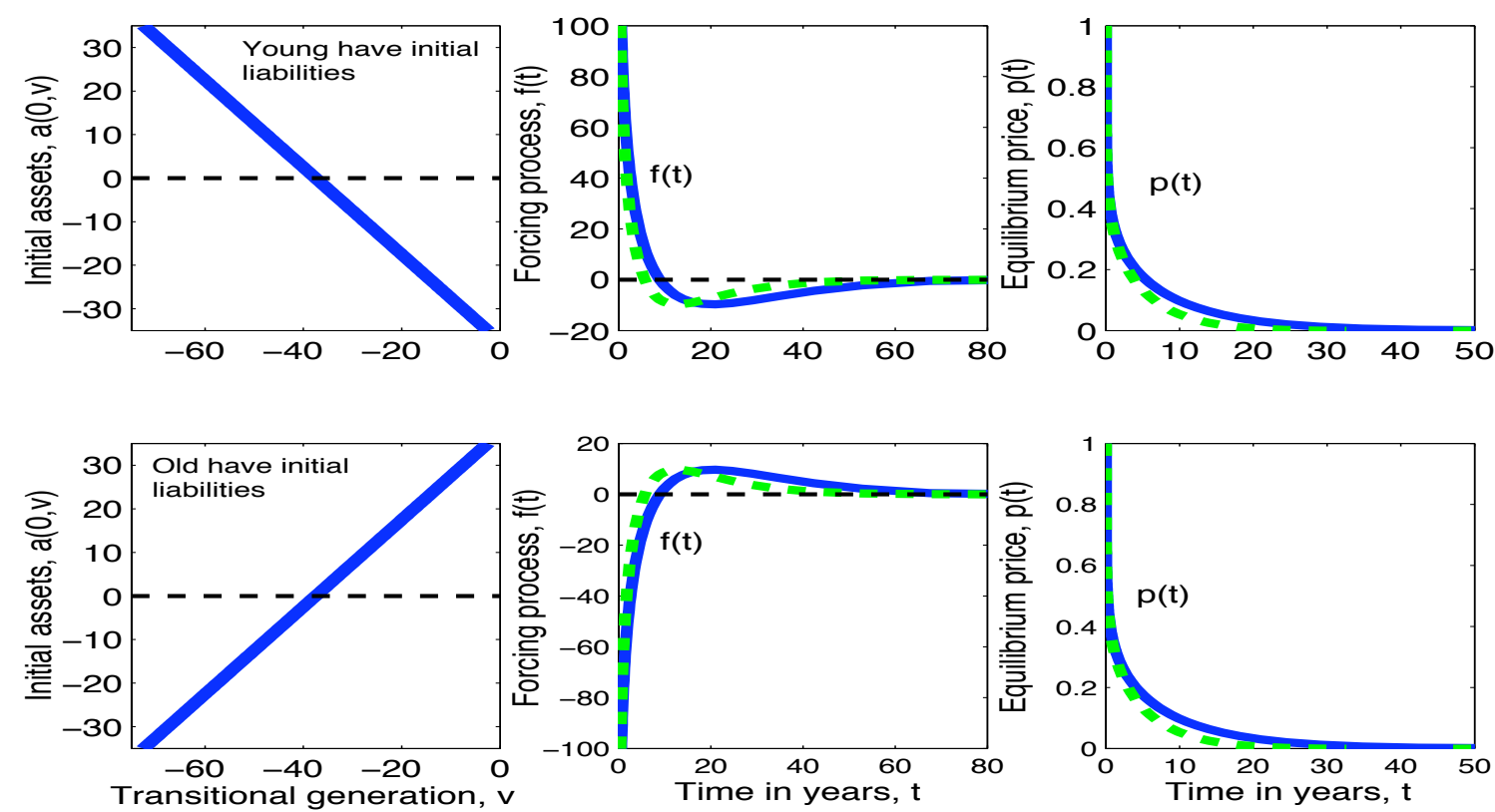

Figure 2: Sensitivity to initial asset distribution $a(0, v)$. Solid lines have discount rate $\rho=0.05$, dashed lines have $\rho=0.10$. Equilibrium prices are much more sensitive to $\rho$ than to the initial distribution. 
To get some sense of how big an influence is exerted by the pattern of individual endowments, consider the bottom panel of Figure 3. This shows the same exercise but with growing endowments $Y(t)=e^{0.05 t}$. Relative to this increase in the aggregate growth rate, the change in the distribution of individual endowments has a large effect on equilibrium prices.
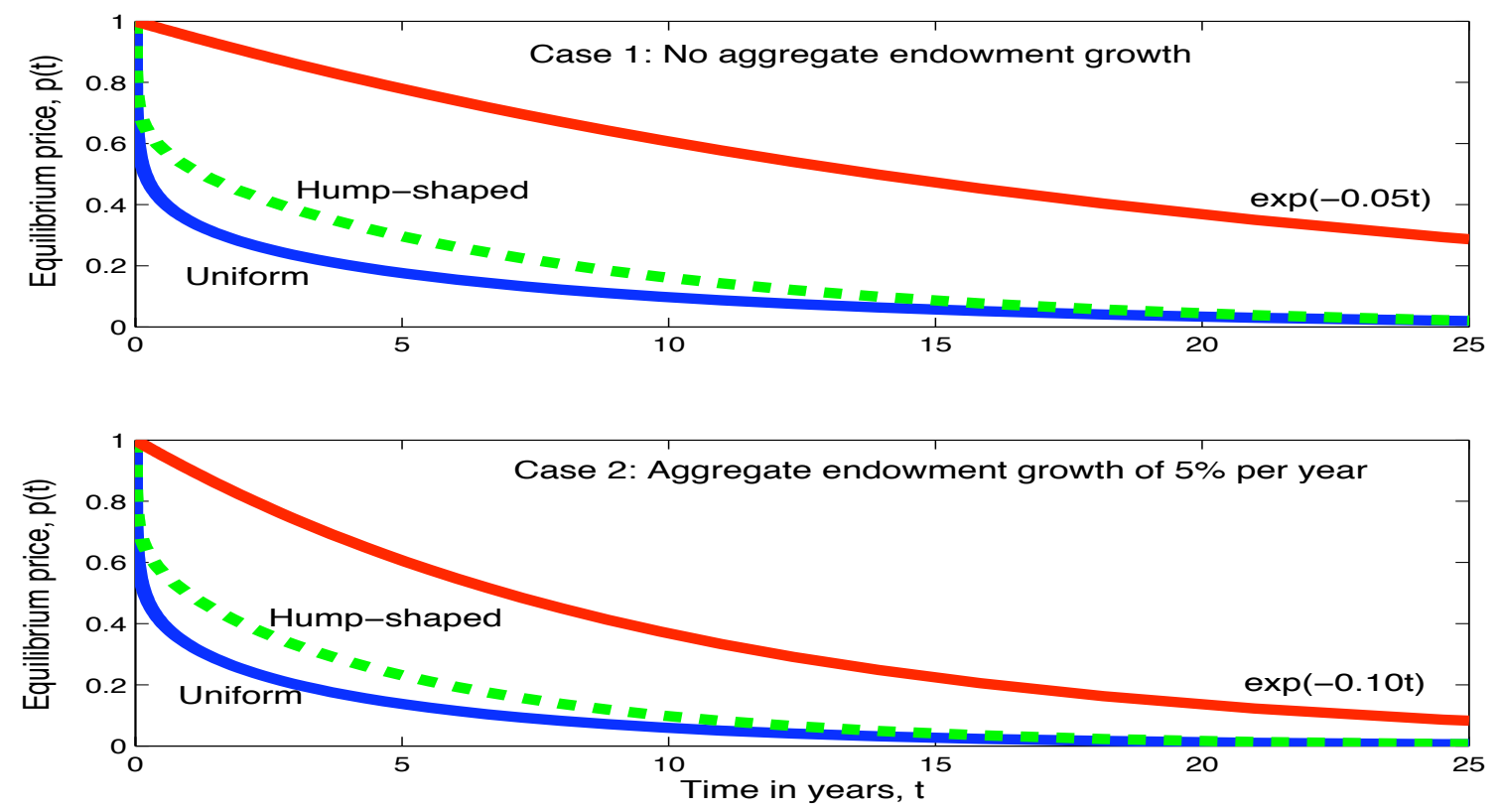

Figure 3: The change in the distribution of individual endowments (from uniform to 'hump-shaped' in age) has a large effect on $p(t)$. The top panel has discount rate $\rho=0.05$ and no aggregate endowment growth $\gamma=0$. The bottom panel has growing aggregate endowment $\gamma=0.05$.

\section{Solving the model with general isoelastic utility}

Recall that we are interested in solutions to the fixed point problem $p=T_{\sigma} p$ where $T_{\sigma}$ is the nonlinear operator:

$$
\left(T_{\sigma} p\right)(t)=\int_{0}^{\infty} p(s) k_{\sigma}(p, t, s) d s+f_{\sigma}(p, t)
$$

Global approximations to the equilibrium price function can be obtained by iterating on an approximating nonlinear operator formed using numerical quadrature. Suppose we have a $J$-point quadrature rule with nodes $t_{i}$ and weights $\omega_{i}$ for $t \in[0, \infty)$. Suppose also that we have some $J$-dimensional candidate price vector $\hat{p}_{n}$ with typical elements $\hat{p}_{n, i}$. For example, this candidate price vector may be the solution from the same model but with log utility.

Now associate with each $t_{i}$ a set of $M$ quadrature nodes $z_{i m}$ and weights $\delta_{i m}$ that give numerical integration over $\left[t_{i}-l, t_{i}\right)$. Write the approximate expenditure shares given the price function $\hat{p}_{n}$ as $\hat{\alpha}_{n}$ with typical elements:

$$
\hat{\alpha}_{n, i m}:=\frac{e^{-\frac{\rho}{\sigma} t_{i}} \hat{p}_{n, i}^{\frac{\sigma-1}{\sigma}}}{\sum_{j=1}^{J} e^{-\frac{\rho}{\sigma} t_{j}} \hat{p}_{n, j}^{\frac{\sigma-1}{\sigma}} \mathbb{1}\left\{t_{j} \in \mathcal{A}\left(z_{i m}\right)\right\} \omega_{j}}
$$


for $i=1, \ldots, J$ and $m=1, \ldots, M$ (in this expression, $\mathbb{1}$ denotes the indicator function). Similarly compute elements $\hat{k}_{n, i j}$ and $\hat{f}_{n, i j}$ according to:

$$
\begin{aligned}
\hat{k}_{n, i j} & :=\sum_{m=1}^{M} \hat{\alpha}_{n, i m} \frac{y\left(t_{j}, z_{i m}\right)}{Y\left(t_{i}\right)} \delta_{i m} \omega_{j} \\
\hat{f}_{n, i} & :=\sum_{m=1}^{M} \hat{\alpha}_{n, i m} \frac{a\left(0, z_{i m}\right)}{Y\left(t_{i}\right)} \delta_{i m}
\end{aligned}
$$

for $i, j=1, \ldots, J$. With these formulas in hand, a new estimate of the price vector, $\hat{p}_{n+1}$ is computed with typical elements given by the formula:

$$
\hat{p}_{n+1, i}=\sum_{j=1}^{J} \hat{p}_{n, j} \hat{k}_{n, i j}+\hat{f}_{n, i}
$$

for $i=1,2, \ldots, J$.

Now test if the norm $\left\|\hat{p}_{n+1}-\hat{p}_{n}\right\|$ meets some pre-set tolerance criterion. If not, update to (say) $\hat{p}_{n+1}$ and compute new expenditure shares, $\hat{\alpha}_{n+1, i m}$, and new coefficients, $\hat{k}_{n+1, i j}$ and $\hat{f}_{n+1, i}$, using (32)-(33). This procedure is repeated until $\left\|\hat{p}_{n+1}-\hat{p}_{n}\right\|$ is sufficiently small.

\section{Related literature}

Work on continuous time OLG economies began with Cass and Yaari (1967). They study a model with finite horizons, log utility and physical capital. They derive the possibility of multiple balanced growth equilibria [analogous to multiple solutions $r$ to equation (24)] and examine the ways in which dynamic efficiency can be addressed in such a setting. OLG models with continuous time but infinite horizons were studied by Blanchard (1985) and Weil (1989). Farmer (2002) uses a stochastic version of Weil's model for business cycle analysis. The tradition of using long-lived discrete time OLG models for policy analysis is due to Auerbach and Kotlikoff (1987). Ríos-Rull (1996) is the standard reference on related long-lived discrete time stochastic OLG economies. Burke (1996) tackled the problem of equilibrium existence in a continuous time OLG setting.

A closely related recent paper is Demichelis and Polemarchakis (2007) who have a continuous time OLG economy with log utility but no discounting and restrictive assumptions about endowments. ${ }^{5}$ Two other closely related papers are d'Albis and Augeraud-Véron (2004, 2007). d'Albis and Augeraud-Véron (2004) has production and log utility but age-independent wages, i.e., every individual alive at $t$ receives the same competitive wage (there is no heterogeneity in the quantity of efficiency units supplied over the life-cycle). Production exhibits constant returns in capital so the real interest rate and hence intertemporal prices are essentially exogenous. They then derive capital accumulation paths consistent with asset market clearing. They study the problem by recasting the integral equation that emerges naturally from market clearing conditions as a delay-differential equation. d'Albis and Augeraud-Véron (2007) studies an exchange economy with age-independent

\footnotetext{
${ }^{5}$ The advantage of these additional assumptions give rise to a linear integral equation of the convolution kind that can (almost) be solved in closed form by Fourier transformation.
} 
endowments but general isoelastic utility. The analysis uses local perturbations to a steady state and ignores transitional generations.

\section{Conclusions}

This paper provides a method for solving for the dynamic general equilibrium of a deterministic continuous time OLG model. The model allows for isoelastic utility and general assumptions about individual endowments (and demographics). The computational task in solving for an equilibrium reduces to solving a specific nonlinear integral equation problem. In the case of log utility the integral equation becomes linear and global approximations can be obtained with finite dimensional linear algebra. The paper outlines a numerical procedure for solving the general integral equation and provides examples.

A continuous time OLG model useful for applied macroeconomics must also be able to handle stochastic environments, capital accumulation and elastic labor supply, amongst other things. Perhaps the framework developed in this paper can be extended along these lines.

\section{A Variable demographics and within-cohort heterogeneity}

The mass of agents of generation $v \in(-l, \infty)$ at date $t \geq 0$ is assumed to be given by a function $n(t, v) \geq 0$. This notation allows for exogenously given changes in the relative proportions of young and old. Let each generation be a continuum $[0,1]$ of individuals indexed by $i$ with idiosyncratic

endowment $y(i, t, v) \geq 0$ at $t$ and aggregate endowment $Y(t):=\int_{0}^{1} \int_{\mathcal{G}(t)} y(i, t, v) n(t, v) d v d i>0$. Let the initial asset distribution be $a(i, 0, v)$ with $\int_{0}^{1} \int_{\mathcal{G}(0)} a(i, 0, v) n(0, v) d v d i=0$. Individual consumption is again given by:

$$
c_{\sigma}(p, i, t, v):=\alpha_{\sigma}(p, t, v) \frac{W(p, i, v)}{p(t)}
$$

where $\alpha_{\sigma}(p, t, v)$ denotes the expenditure shares, the same as in equation (8), and where individual intertemporal wealth is:

$$
W(p, i, v):=\int_{0}^{\infty} p(s) y(i, s, v) d s+p(0) a(i, 0, v)
$$

Market clearing requires $\int_{0}^{1} \int_{\mathcal{G}(t)} c_{\sigma}(p, i, t, v) n(t, v) d v d i=Y(t)$. This can be rearranged to give the Urysohn integral equation $p(t)=\int_{0}^{\infty} p(s) k_{\sigma}(p, s, t) d s+f_{\sigma}(p, t)$ as in equation (10) but now:

$$
\begin{aligned}
k_{\sigma}(p, t, s) & =\int_{0}^{1} \int_{\mathcal{G}(t)} \alpha_{\sigma}(p, t, v) \frac{y(i, s, v)}{Y(t)} n(t, v) d v d i \\
f_{\sigma}(p, t) & =\int_{0}^{1} \int_{\mathcal{G}(t)} \alpha_{\sigma}(p, t, v) \frac{a(i, 0, v)}{Y(t)} n(t, v) d v d i
\end{aligned}
$$

This allows for general endowments and demographics but the analysis is the same.

Almost immediately this allows the model to be generalized to an economy with deterministic aggregate endowment but stochastic idiosyncratic endowments that can be insured away by dynamically complete markets (in which case equilibrium individual consumption is deterministic). 


\section{References}

Atkinson, K. E. (1997): The numerical solution of integral equations of the second kind. Cambridge University Press. 2, 4, 6, 8

Auerbach, A. J., And L. J. Kotlikoff (1987): Dynamic fiscal policy. Cambridge University Press. 1, 12

BAKeR, C. T. (1977): The numerical treatment of integral equations. Clarendon Press. 2, 5, 6, 8

Blanchard, O. J. (1985): "Debt, deficits, and finite horizons," Journal of Political Economy, 93(2), 223-247. 1, 4, 12

Burke, J. L. (1996): "Equilibrium for overlapping generations in continuous time," Journal of Economic Theory, 70, 346-390. 12

CASs, D., AND M. YAARI (1967): "Individual savings, aggregate capital accumulation and efficient growth," in Essays on the theory of optimal economic growth, ed. by K. Shell. MIT Press. 12

D'Albis, H., And E. Augeraud-VÉron (2004): "Competitive growth in a life-cycle model: Existence and dynamics," EUREQua Université Paris-I Panthéon-Sorbonne working paper. 12 (2007): "Balanced cycles in an OLG model with a continuum of finitely-lived individuals," Economic Theory, 30(1), 181-186. 12

Demichelis, S., And H. M. Polemarchakis (2007): "The determinacy of equilibrium in economies of overlapping generations," Economic Theory, 32(3), 461-475. 12

Diamond, P. A. (1965): "National debt in a neoclassical growth model," American Economic Review, 55(5), 1126-1150. 2

FARMer, R. E. (2002): "Business cycles with heterogenous agents," UCLA working paper. 12

JuDD, K. L. (1998): Numerical methods in economics. MIT Press. 2, 6, 8

LuCAS, R. E. (1978): "Asset prices in an exchange economy," Econometrica, 46(6), 1429-1445. 6

Press, W. H., S. A. Teukolsky, W. T. Vetterling, and B. P. Flannery (1992): Numerical recipes in $C$ : The art of scientific computing. Cambridge University Press. 2, 4, 6, 8

Ríos-Rull, J.-V. (1996): "Life-cycle economies and aggregate fluctuations," Review of Economic Studies, 63(3), 465-489. 1, 12

Tauchen, G., And R. Hussey (1991): "Quadrature-based methods for obtaining approximate solutions to nonlinear asset pricing models," Econometrica, 59(2), 371-396. 6

Tricomi, F. (1957): Integral equations. Dover. 6

WeIL, P. (1989): "Overlapping families of infinitely-lived agents," Journal of Public Economics, 38(2), 183-198. 1, 4, 12

YAARI, M. E. (1965): "Uncertain lifetime, life insurance, and the theory of the consumer," Review of Economic Studies, 32(2), 137-150. 1 